\title{
Payer Coverage for Hereditary Cancer Panels: Barriers, Opportunities, and Implications for the Precision Medicine Initiative
}

\author{
Julia R. Trosman, PhDª, ; Christine B. Weldon, MBA, \\ Allison W. Kurian, MD, MSc ${ }^{\text {; }}$ R. Kate Kelley, MD ${ }^{a, e, f}$; Patricia A. Deverka, MD, MSg; \\ and Kathryn A. Phillips, PhD ${ }^{\mathrm{a}, \mathrm{f}, \mathrm{h}}$
}

\begin{abstract}
Background: Hereditary cancer panels (HCPs), testing for multiple genes and syndromes, are rapidly transforming cancer risk assessment but are controversial and lack formal insurance coverage. We aimed to identify payers' perspectives on barriers to HCP coverage and opportunities to address them. Comprehensive cancer risk assessment is highly relevant to the Precision Medicine Initiative (PMI), and payers' considerations could inform PMI's efforts. We describe our findings and discuss them in the context of PMI priorities. Methods: We conducted semi-structured interviews with 11 major US payers, covering > 160 million lives. We used the framework approach of qualitative research to design, conduct, and analyze interviews, and used simple frequencies to further describe findings. Results: Barriers to HCP coverage included poor fit with coverage frameworks (100\%); insufficient evidence (100\%); departure from pedigree/family history-based testing toward genetic screening (91\%); lacking rigor in the HCP hybrid research/clinical setting (82\%); and patient transparency and involvement concerns (82\%). Addressing barriers requires refining HCP-indicated populations ( $82 \%)$; developing evidence of actionability $(82 \%)$ and pathogenicity/penetrance (64\%); creating infrastructure and standards for informing and recontacting patients (45\%); separating research from clinical use in the hybrid clinical-research setting (44\%); and adjusting coverage frameworks (18\%). Conclusions: Leveraging opportunities suggested by payers to address HCP coverage barriers is essential to ensure patients' access to evolving HCPs. Our findings inform 3 areas of the PMI: addressing insurance coverage to secure access to future PMI discoveries; incorporating payers' evidentiary requirements into PMI's research agenda; and leveraging payers' recommendations and experience to keep patients informed and involved.
\end{abstract}

\section{Background}

Identification of hereditary cancer predisposition is an important component of cancer risk management, prevention, and treatment. ${ }^{1-3}$ Its significance was underscored recently by President Obama's Precision

From aUCSF Center for Translational and Policy Research on Personalized Medicine (TRANSPERS), Department of Clinical Pharmacy, University of California, San Francisco, San Franscisco, California; ${ }^{b}$ Center for Business Models in Healthcare, Chicago, Illinois; 'Feinberg School of Medicine, Northwestern University, Chicago, Illinois; 'Departments of Medicine and of Health Research and Policy, Stanford University School of Medicine, Stanford, California; eDepartment of Medicine, Division of Hematology/ Oncology, University of California, San Francisco, San Francisco, Califorina; ${ }^{f}$ Helen Diller Family Comprehensive Cancer Center, University of California, San Francisco, San Francisco, California; 'American Institutes for Research, Chapel Hill, North Carolina; and hPhilip R. Lee Institute for Health Policy, University of California, San Francisco, San Francisco, California.

Submitted May 10, 2016; accepted for publication November 8, 2016.
Medicine Initiative (PMI) announced in 2015, 4,5 with objectives that include advancing inherited cancer genomics. ${ }^{6-8}$ The advent of hereditary cancer panels (HCPs)_defined here as multigene, multisyndrome, next-generation sequencing panels for hereditary can-

Dr. Kurian has disclosed that she has received research funding from Myriad Genetics, Invitae, and Ambry Genetics. The remaining authors have disclosed that they have no financial interests, arrangements, affiliations, or commercial interests with the manufacturers of any products discussed in this article or their competitors.

Author Contributions: Conception and design: Trosman, Weldon, Phillips. Collection and assembly of data: Trosman, Weldon, Phillips. Data analysis and interpretation: Trosman, Weldon, Douglas, Kurian, Kelley, Deverka, Phillips. Manuscript writing: Trosman, Weldon, Douglas, Kurian, Kelley, Deverka, Phillips. Final approval of manuscript: Trosman, Weldon, Douglas, Kurian, Kelley, Deverka, Phillips.

Correspondence: Julia R. Trosman, PhD, Center for Business Models in Healthcare, 2705 West Agatite, Suite 200, Chicago, IL 60625.

E-mail: trosman@centerforbusinessmodels.com 
Trosman et al

cer testing containing well-studied and less-studied genes-is reshaping clinical cancer risk assessment and igniting cancer genetics research. ${ }^{9-13}$ HCPs represent a transitional step between traditional singlegene/single-syndrome testing and whole-genome/ exome sequencing (WGS/WES), ${ }^{14-16}$ and some of the advantages of HCPs serve as a proxy for those of WGS/WES, including more comprehensive and rapid testing than step-wise single-gene assessment, identification of risk not suggested by pedigree, and accelerated data collection for research. ${ }^{12,13,15-17}$ However, the clinical appropriateness of using HCPs instead of pedigree- or phenotype-directed single-gene tests is hotly debated ${ }^{17-19}$; guidelines recommend caution ${ }^{1,2}$ and some experts consider their broadening clinical use premature. ${ }^{14,18,20,21}$ Nevertheless, HCP commercial offerings proliferate $e^{9,17,21}$ and their increasing clinical adoption is viewed by some as "the train that's left the station."

Adding to the controversy is the complexity of HCPs' insurance coverage and reimbursement. Although HCP providers may receive ad hoc payment (eg, when billing with generic or single-gene test codes, or on appeal ${ }^{22,23}$ ), payers don't formally cover HCPs. ${ }^{23-25}$ Our previous review of the 17 largest US private payers' policies for HCPs containing BRCA1/2 genes found no positive coverage as of May 2015.22 The absence of formal coverage causes reimbursement inconsistency and uncertainty, impacts patient access, and contributes to practice heterogeneity. Indicating a continued lack of coverage, our more recent data for 5 of the largest private payers from the University of California, San Francisco (UCSF) TRANSPERS Payer Coverage Policy Registry ${ }^{26}$ showed no or substantially limited HCP coverage (see supplemental eAppendix 1, available with this article at INCCN.org). These and other data noted earlier motivated the present study, for which the objective is to elucidate payers' barriers to HCP coverage and opportunities to address them (the 5 payers in supplemental eAppendix 1 are part of the 11 -payer cohort in the present study).

Understanding payers' coverage decision-making is critical for clarifying the HCP reimbursement state for patients and physicians, informing genetic research, and ultimately enabling access to these potentially transformative modalities. Barriers to HCP coverage foreshadow those of WGS/WES, and ad- dressing them may facilitate future access to WGS/ WES as science evolves. Further, payers' HCP coverage considerations have particular relevance to the PMI and its effort to assemble and study an unprecedented cohort of $\geq 1$ million volunteers (PMI cohort, or the All of Us Research Program) who will contribute genomic, clinical, and lifestyle data to accelerate genetic science. ${ }^{4,5,7}$ Incorporation of increasingly comprehensive genomic testing into our understanding of cancer risk and approaches to treatment is integral to the PMI, ${ }^{27}$ which will likely contribute to further development of multigene testing technologies. Thus, challenges to insurance coverage of these technologies must be understood and addressed proactively to ensure effective translation of future PMI results into care. Payer evidentiary requirements for HCPs could inform PMI's research agenda and increase future relevance of research outcomes to payers' coverage decisions. This article describes our study findings and discusses their implications for the PMI.

\section{Methods}

The study was approved by the UCSF Institutional Review Board. We adapted the framework approach of qualitative research ${ }^{28,29}$ for study design and data analysis as an effective method for eliciting stakeholders' perspectives (including payers and clinical experts) and examining coverage issues, previously used by us and other researchers. ${ }^{30-37}$

We first conducted semi-structured interviews with 12 clinical experts possessing direct knowledge of and experience in HCP research and clinical applications; development of the expert interview guide (supplemental eAppendix 2) was informed by literature review. The interviews focused on identifying characteristic HCP features, HCP advantages compared with single-gene testing, and HCP controversies and challenges (supplemental eAppendix 2). Phone interviews took 45 to 55 minutes each and were taped, transcribed, and analyzed for common themes, according to the framework approach, by 2 independent investigators. Discrepancies were resolved by discussion and consensus. Resulting themes were summarized into an HCP Case Study (Table 1), and helped formulate the structure and content of the payer interview questions (supplemental eAppendix 2). 
Coverage for Hereditary Cancer Panels

\begin{tabular}{|c|c|}
\hline HCPb Characteristics & Description \\
\hline \multicolumn{2}{|l|}{ HCP features ${ }^{9,10,15,21,22}$} \\
\hline \multirow[t]{2}{*}{ Disease scope } & $\begin{array}{l}\text { One cancer, multiple syndromes (eg, panels for hereditary breast cancer, testing for HBOC, } \\
\text { Li-Fraumeni, Cowden, and Peutz-Jeghers syndromes) }\end{array}$ \\
\hline & Multiple cancers, multiple syndromes \\
\hline Number of genes & Range from 6 to $>100$ genes \\
\hline \multirow[t]{2}{*}{ Familiarity of genes } & $\begin{array}{l}\text { Well-studied genes: high familiarity and evidence on pathogenicity, cancer risk, and/or intervention } \\
\text { outcomes (eg, BRCA1/2) }\end{array}$ \\
\hline & Less-studied genes: lower familiarity, less evidence on pathogenicity, and/or risk level \\
\hline \multirow[t]{3}{*}{ Testing results } & Pathogenic variant: results in a dysfunctional protein, consistent with a disease phenotype \\
\hline & $\begin{array}{l}\text { VUS: sequence variant (usually subtle, such as a single nucleotide polymorphism or other missense } \\
\text { change) that may or may not result in a dysfunctional protein and a disease phenotype }\end{array}$ \\
\hline & Normal: no variation from normal sequence \\
\hline \multirow[t]{2}{*}{ Gene penetrance } & High penetrance: alterations confer high lifetime risk of cancer $(40 \%-80 \%)$ \\
\hline & Medium penetrance: alterations confer medium lifetime risk of cancer $(20 \%-40 \%)$ \\
\hline \multicolumn{2}{|l|}{ HCP advantages vs single gene testing ${ }^{1,10,13,17,22}$} \\
\hline \multirow{2}{*}{$\begin{array}{l}\text { Address syndrome heterogeneity and } \\
\text { overlaps }\end{array}$} & Increasing understanding that pedigree may not be suggestive of particular alterations \\
\hline & HCPs identify alterations that would not have been evaluated in phenotype-directed testing \\
\hline Testing efficiency effectiveness & $\begin{array}{l}\text { Streamlines testing compared to step-wise, single-gene testing; prevents testing fatigue for patients } \\
\text { and clinicians; sequences more genes for comparable cost }\end{array}$ \\
\hline \multirow[t]{2}{*}{$\begin{array}{l}\text { Accelerate research in a hybrid } \\
\text { research/clinical setting }\end{array}$} & $\begin{array}{l}\text { The setting of combined research and clinical use is emerging, resembling the evolution of clinical } \\
\text { BRCA1/2 testing }\end{array}$ \\
\hline & $\begin{array}{l}\text { Considered an effective way to advance research on less-studied genes and reclassify VUS to clinically } \\
\text { useful results, which would otherwise take decades and vast patient cohorts }\end{array}$ \\
\hline $\begin{array}{l}\text { Expedite application of future } \\
\text { research }\end{array}$ & $\begin{array}{l}\text { Inclusion of less-studied genes allows to expediently identify and recontact relevant patients when } \\
\text { new evidence becomes available }\end{array}$ \\
\hline \multicolumn{2}{|l|}{ HCP controversies and challenges ${ }^{1,9-11,16,18,20}$} \\
\hline $\begin{array}{l}\text { Debate on how to determine } \\
\text { actionability }\end{array}$ & $\begin{array}{l}\text { Should management of less-studied alterations with moderate to high cancer risk (1) be similar to } \\
\text { that of well-studied alterations or (2) be studied to prove outcomes? }\end{array}$ \\
\hline $\begin{array}{l}\text { Require a different genetic counseling } \\
\text { model }\end{array}$ & $\begin{array}{l}\text { HCPs require extensive pretest and posttest genetic counseling, but the current counseling paradigm } \\
\text { based on single-gene testing is not adequate for HCPs }\end{array}$ \\
\hline $\begin{array}{l}\text { Emerging state of evidence and } \\
\text { guidelines }\end{array}$ & $\begin{array}{l}\text { Scarce validity and utility/actionability data for many HCP genes, and lack of consistency and clarity in } \\
\text { guidelines have not prevented HCP clinical adoption }\end{array}$ \\
\hline
\end{tabular}

Abbreviations: HBOC, hereditary breast and ovarian cancer syndrome; HCP, hereditary cancer panel; VUS, variant of uncertain significance. aThis case study describes illustrative features, advantages, and challenges of HCPs and does not represent a comprehensive analysis of HCPs. ${ }^{b} \mathrm{HCPs}$ are defined here as next-generation sequencing assays that simultaneously test multiple genes for susceptibility to multiple cancer syndromes, sequence both well-studied and less-studied genes, include high and moderate penetrance alterations, and return VUS.

Subsequently, semi-structured interviews were conducted with 11 private US payers. We focused the study on private payers because they collectively cover nearly two-thirds of the insured US population, ${ }^{38}$ and their policies impact the public health plans they manage, such as some state Medicaid and Medicare Advantage programs. ${ }^{39}$

We used purposive sampling ${ }^{40}$ to assemble the payer and clinical expert interview cohorts, both recruited via the UCSF TRANSPERS Evidence and Reimbursement Policy Advisory Council. All member-payers chose to participate in the study. The payer cohort included senior executives with coverage decision-making responsibilities from the 8 largest US private payers and 3 regional payers, collectively covering $>160$ million enrollees. ${ }^{41}$

An interview guide, including the HCP Case Study (Table 1) and interview questions (supplemental eAppendix 2), was provided before the interviews. The semi-structured phone interviews with payers were conducted between February and June 2015. Two investigators performed independent thematic coding and analysis, resolving disagreement by discussion and consensus. We used simple frequencies to further describe identified themes. 
Trosman et al

\section{Results}

Payer interviews revealed a range of themes reflecting payers' perspectives on barriers to positive HCP coverage and suggested opportunities to overcome them. The following sections describe the findings by categories of barriers and opportunities (summarized in Table 2).

\section{HCPs Do Not Fit Payer Coverage Frameworks}

Most payers (73\%) stated that they understood why novel HCP features present unique appeal to clinicians and patients. Payers recognized that the inclusion of less-studied genes in panels promotes research and helps future dissemination of new genetic knowledge via patient recontacting (64\%). They also acknowledged limitations in our current understanding of phenotype/genotype associations and agreed that multisyndrome testing may identify alterations unsuspected from pedigree (73\%). However, interviewees explained that these very features make positive coverage challenging, because they do not fit exist- ing coverage frameworks. Because HCPs include both well-studied ("medically necessary") and less-studied ("experimental/investigational") genes, they don't fit either category; thus, an entire panel is deemed experimental. Although all payers noted this barrier, only $18 \%$ believed that coverage frameworks should be modified to align with panel features and benefits. Three other payers $(27 \%)$ contemplated potential approaches to HCP coverage, including separating billing/coding for medically necessary from experimental/ investigational genes in one $\mathrm{HCP}$ and paying only for those medically necessary, and/or requiring that a test provider establish a registry studying experimental/investigational genes within a panel, with demonstrated research rigor and data-sharing practices.

Similarly, interviewees described that the departure from phenotype-directed testing conflicts with the current concept of predefined medical necessity of specific suspected genes, and will likely lead to indiscriminant genetic screening (91\%). However, they signaled their willingness to expand testing populations and relax testing criteria if evidence of

Table 2. Payers' Perspectives on Barriers to HCP Insurance Coverage and Opportunities to Address Barriers

\begin{tabular}{|ll}
$\begin{array}{l}\text { Barrier Categories; } \\
\text { \% of Payers Noting } \\
\text { at Least One Challenge } \\
\text { (N=11) }\end{array}$ & $\begin{array}{l}\text { Payers' Description of Specific } \\
\text { Barriers (N=11) }\end{array}$ \\
\hline $\begin{array}{l}\text { HCPs don't fit payers' } \\
\text { coverage frameworks } \\
(100 \%)\end{array}$ & $\begin{array}{l}\text { HCPs don't fit definitions of } \\
\text { medically necessary" and } \\
\text { "investigational" (100\%) }\end{array}$ \\
\cline { 2 - 2 } $\begin{array}{l}\text { Gaps in evidence (100\%) } \\
\text { HCPs move genetic testing toward } \\
\text { preselected populations (91\%) }\end{array}$ & $\begin{array}{l}\text { Lacking evidence of pathogenic } \\
\text { significance and penetrance of } \\
\text { many variants in panels (100\%) }\end{array}$ \\
\hline $\begin{array}{l}\text { mybrid research/clinical } \\
\text { setting for HCPs is not } \\
\text { acceptable by payers (91\%) }\end{array}$ & $\begin{array}{l}\text { Lacking definition and data on } \\
\text { transparency (82\%) }\end{array}$ \\
$\begin{array}{l}\text { actionability of cancer genetic } \\
\text { alterations (82\%) }\end{array}$ \\
\hline $\begin{array}{l}\text { Hybrid means de facto coverage/ } \\
\text { reimbursement for research (73\%) }\end{array}$ \\
\hline HCP testing (82\%) & $\begin{array}{l}\text { Lack of transparency to patients } \\
\text { (73\%) }\end{array}$ \\
\hline
\end{tabular}

HCP testing $(82 \%)$

Payer Recommendations to Address Barriers ( $\mathrm{N}=11)$

Align the coverage frameworks to enable evaluation of new tests, such as HCPs $(18 \%)$

Adjust the coverage frameworks to account for non-direct clinical benefits, such as ease of use (18\%)

Prove existence and prevalence of syndrome/gene heterogeneity $(73 \%)$

Better define subpopulations indicated for HCP to avoid universal screening and develop panels for these subpopulations ( $82 \%)$

Must have evidence of significance and penetrance for all genes on an HCP $(64 \%)$

Develop standards and decision tools for patients and clinicians to deal with less proven variants in HCPs $(27 \%)$

Define criteria when penetrance data are sufficient, and when outcomes data are needed $(36 \%)$

Evidence of penetrance is sufficient to determine actionability (27\%)

Develop a novel, rigorous, transparent model for the hybrid approach, delineating research and standard care $(44 \%)$

Hybrid should separate funding of research vs standard care (36\%)

Increase transparency to patients on the state of evidence for HCPs and related interventions $(73 \%)$

Capture and accommodate patient preferences for testing and recontacting (45\%) Determine patient utility of HCPs via patient reported outcomes research (36\%)

Patient recontacting with new Establish and standardize process, infrastructure, and accountability for data is ad hoc, not standard (64\%) recontacting (45\%)

Abbreviation: HCPs, hereditary cancer panels. 
syndrome heterogeneity were provided (73\%), and the relevant populations were more precisely defined to avoid "universal testing" (82\%). Payers noted specific end points for these evidentiary requirements, including identifying which syndromes overlap and in what combinations; determining prevalence of overlaps compared with single-syndrome prevalence; and elucidating characteristics of populations with overlaps to indicate appropriateness of panel testing.

\section{Gaps in Evidence on HCPs, and Views on Genetic Alteration Actionability}

All interviewees stated that a major impediment to HCP coverage is insufficient evidence on lessstudied genes, specifically evidence of clinical validity with respect to pathogenicity (association with a cancer syndrome) and penetrance (degree of cancer risk). Although most payers (64\%) would require this evidence for all genes in an HCP, a minority $(27 \%)$ stated they could cover panels with lessstudied genes if specific protocols and tools were developed for patients/families and clinicians to guide posttest decisions and prevent unnecessary testing, interventions, and anxiety.

Most payers $(82 \%)$ also noted the scarcity of data on HCP clinical utility, particularly actionability of alterations in less-studied genes. However, their opinions varied on how actionability should be defined, specifically, (1) whether management of alterations in less-studied genes conferring moderate to high cancer risk should be similar to that of alterations in well-understood genes (eg, BRCA1/2), or (2) whether management strategies should be studied for alterations in each newly tested gene to prove their effectiveness. Only a minority of payers (36\%) would require that the outcomes of interventions for alterations in each new gene be studied if these interventions are already proven for alterations in other genes related to the same syndrome. Conversely, other payers stated that outcomes studies of alterations in each newly tested gene are not necessary: 27\% regarded the "similar management" approach as reasonable, whereas another 36\% suggested a mixed model, which would require outcome studies for some but not all genes, based on "plausibility of similarity in outcomes with alterations in known genes" and on syndrome prevalence. Lack of agreement between medical societies on how to de- fine an actionable mutation was noted as a barrier by $82 \%$ of interviewees.

Nine payers $(82 \%)$ shared perspectives on what study designs may constitute evidence acceptable for HCP coverage (data are not shown in Table 2). They noted that randomized control trials may not be required; observational studies, registries, and pooled studies could be acceptable, as long as they are large enough to demonstrate statistical power and significance of findings. A total of $18 \%$ would require at least one additional study to confirm original findings, citing particular importance of the "do no harm" in asymptomatic populations. Although $82 \%$ of payers would require the studies to be published in peer-reviewed journals, they did not share preferences for particular journals, and 27\% would take guidance from medical societies to assess reputability of published results.

\section{HCPs' Hybrid Clinical/Research Setting is Concerning}

Payers recognized that HCPs are used in a hybrid research/clinical setting, in which increasing $\mathrm{HCP}$ clinical use generates data to elucidate validity and utility of less-studied genes, in turn enhancing HCP clinical use. Interviewees acknowledged that this approach may accelerate data collection and that it enabled the past evolution of BRCA1/2 testing. Nonetheless, payers considered the hybrid setting inappropriate as either a clinical or a research model $(91 \%)$, because as a clinical setting, it uses unproven testing, and as a research setting, it lacks rigor and transparency, putting resulting data into question (82\%). Additionally, $73 \%$ of interviewees were concerned that if they cover HCP clinical use, they will be paying for research in the hybrid setting. They commented that financing research, either by covering experimental technologies or by direct funding, conflicts with payers' mandate to cover only nonexperimental technologies, causes objection from employers (customers of the payers), and confers legal implications. However, interviewees understood that traditionally designed research studies in asymptomatic patients may require decades and large patient cohorts, and realized that the research paradigm is changing. Therefore, they may be willing to support the hybrid HCP setting if it is transformed to a more rigorous, transparent model clearly delineating research from standard care (45\%). Additionally, payers shared how they could support research with- 
out directly funding it, including (1) obtaining grants from payer company foundations; (2) paying for standard care within a research study, as required by the Affordable Care Act; (3) encouraging collaborations between researchers and payers' clinical analysts to develop combined data sets and shorten the research cycle; and (4) identifying large self-insured employers who may be willing to work with a payer to underwrite a research study, if interested in HCPs for their employee base.

\section{Concerns About Patients' Understanding and Engagement}

Most interviewees (82\%) expressed concerns about the impact of HCP testing on patients, and stated that these concerns influenced their coverage decisions. They believed that despite informed consent and genetic counseling, patients may not understand the immature state of evidence about numerous genes within panels $(73 \%)$ and that their data are used for research, to which many would object if better informed. Payers' suspicion that many patients do not receive pretest and/or posttest genetic counseling exacerbated these concerns.

In payers' opinions, the merit of hereditary panel testing may be overpromised to patients by clinicians and test providers. One interviewee noted, "Genetics is met with breathless excitement that's not backed by solid evidence, and not demystified for patients." One such unfounded promise is to capture less-understood alterations now, and later, when more definitive knowledge about their significance becomes available, recontact patients with relevant alterations and offer medical management without retesting. Although $73 \%$ of payers agreed that this may be beneficial, 64\% were skeptical about the feasibility of fulfilling this promise and about variability of recontacting practices across clinicians and genetics laboratories. Addressing these issues will require creating an industry-wide secure recontacting infrastructure, developing standard evidence thresholds and analytics for recontacting, and establishing approaches to honoring patients' data storage and recontacting preferences.

\section{Discussion}

This study examined private payers' perspectives on barriers and opportunities for insurance coverage of
HCPs. Identified barriers included poor fit of HCPs with coverage frameworks, evidence gaps on cancer risk and actionability for numerous genes in panels, insufficient rigor of the HCP's hybrid clinical/research setting, and concerns about appropriate patient transparency and involvement in HCP testing. Payers also shared opportunities to overcome barriers, suggesting evidence that could both meet coverage requirements and be feasible to obtain, outlining improvements to the hybrid HCP setting and approaches to informing patients, and even indicating a possibility of adjusting the diagnostic coverage frameworks. This work builds on our previous study on challenges to coverage for tumor sequencing, ${ }^{34}$ and expands prior findings by identifying coverage barriers unique to HCPs and providing payers' perspectives on potential solutions. This section discusses the study's relevance to the present and future of HCP and cancer multigene testing. We also discuss implications for the PMI, because of its paramount importance, breadth, and potential to frame solutions to evidentiary and policy challenges, including those of HCPs.

\section{Implications for Practices and Reimbursement of Cancer Multigene Testing: Present and Future}

The current HCP reimbursement is complex and confusing for clinicians and patients: although payers formally do not cover HCPs, they may still pay for them, depending on how HCPs are billed or whether they are appealed. 22,23 This creates payment uncertainty among payers and among patients within one payer, impeding the ability of medical institutions to develop consistent ordering and clinical protocols and exacerbating heterogeneity of related clinical decisions and practices. Further, variability of HCP reimbursement may increase already existing disparities in access to cancer genetic testing, ${ }^{42-44}$ particularly for medically and socioeconomically vulnerable patients unable to pay out-of-pocket for the more contemporary multigene tests. Our study's findings clarify the confusing HCP reimbursement environment and could help medical institutions' shorterterm efforts to reduce the exposure to reimbursement uncertainty and address the challenges described. For instance, understanding payers' reasons for the lack of HCP coverage could inform clinicians' appeal approaches, and recognizing HCP clinical applications potentially acceptable by payers may inform insti- 
tutional criteria for HCP use (eg, for patients with multicancer phenotype).

To achieve long-term sustainable coverage and reimbursement, concerted multi-stakeholder efforts are needed, especially as multigene testing expands from HCPs to WGS/WES. Because HCP and WGS/ WES share key features, such as inclusion of wellstudied and less-studied genes and testing not indicated by pedigree, they may also encounter similar challenges. ${ }^{14-16}$ In fact, our findings suggest that HCP coverage challenges may also indicate barriers to coverage for WGS/WES. Of particular importance is the requirement of coverage frameworks that all genes within a test be medically necessary, which may deem WGS/WES noncoverage permanent. Indeed, a CMS official noted that WGS is "something that CMS would never cover," ${ }^{45}$ later amending this remark to state that it would be "very, very challenging" to collect convincing data. ${ }^{46}$ However, several payers in our study signaled willingness to adjust coverage frameworks for multigene testing, and suggested specific approaches to evidence development and potential HCP coverage. This may serve as a start of a broader multi-stakeholder dialogue and policy effort to facilitate evidence generation and update assessment, coverage, and reimbursement approaches for HCPs, and ultimately for WGS/WES.

\section{Implications for the PMI}

Addressing HCP Insurance Coverage: The PMI's vision includes developing policy pathways to support PMI-based research and translation into care, with an emphasis on modernizing the regulatory evaluation and approval of genomic sequencing technologies. ${ }^{4,5,8}$ Payer coverage for sequencing tests, including HCPs, should also be addressed as a critical link in the precision medicine policy pathway. Shorter-term, positive coverage may enable generation and availability of genomic data to be contributed by the PMI cohort (in addition to data from sequencing for some cohort participants, as being planned to be potentially paid by the $\mathrm{PMI}^{27}$ ), whereas the lack of coverage may considerably limit this opportunity. Longer-term, payer coverage will be a key factor in access to modalities developed by the PMI. Additionally, according to the PMI's vision, new genetic results will be shared with cohort participants who are expected to discuss implications of results, potential further tests, and interventions with their physicians. ${ }^{27}$ Lack of insurance coverage for these tests and interventions will create a conundrum for patients, clinicians, payers, and the PMI.

Thus, addressing the barriers of coverage policy may be as crucial to the PMI's success as optimizing the regulatory policy. Notably, genomic sequencing challenges the frameworks of payer coverage and regulatory policy in similar ways. Our findings indicate that sequencing tests (exemplified by HCPs), which report multiple genetic results that are not defined in advance of testing and vary in the strength of evidence, often do not fit payers' frameworks of medical necessity for coverage. The FDA reported, and experts noted, that these features also strain its regulatory methodology, which was developed for traditional single-gene tests capturing predefined data points anticipated before testing. ${ }^{47-49}$ This commonality provides an opportunity for expanding the PMI's policy scope to include payer coverage and developing a comprehensive policy framework suitable to evaluate and approve genomic sequencing tests from the regulatory and coverage perspectives. Importantly, the PMI calls for modernization of the regulatory framework for genomic sequencing. Whether coverage policy frameworks require modernization should be debated, and at least 3 major payers in our study signaled their openness to a dialogue.

\section{Informing PMI Research Priorities and Methods:}

Both payers and regulators evaluate evidence of analytic and clinical validity for genomic tests, ${ }^{23,30,35,50-52}$ and generating validity data is one of the PMI's objectives. ${ }^{27}$ However, payers also require evidence of clinical utility ${ }^{23,34}$ - how effective and actionable genetic results are in care decisions and interventions — which is beyond the regulatory domain and the current PMI scope. Proving clinical utility of genetic tests to payers has been challenging, partly because of variation in payers' definitions of utility and expectations about the quality of relevant studies. ${ }^{23,30,34} \mathrm{We}$ found that HCPs are no exception, in that payers' evidence requirements for actionability differ, and evidence developed in a hybrid clinical/research setting may not meet the required rigor. Including studies of clinical utility/actionability of genomic testing within the PMI's priorities and considering payers' methodological concerns when designing the PMI Cohort studies would produce the evidence required by payers and accelerate coverage of PMI-derivative tests. However, a consensus across medical societies and payers should 
Trosman et al

be reached first on what constitutes utility and actionability. Although this may be a difficult task, some collaborative efforts have produced cross-payer recommendations for evidence and coverage of genomics, such as tumor sequencing. ${ }^{53}$ The PMI has the scale, authority, and clout to reach this consensus in areas relevant to its priorities.

Enhancing PMI Approaches for Participant Involvement and Transparency: Informed and engaged participants are a hallmark of the PMI cohort. ${ }^{6,8,27,54}$ Participants' involvement will reflect their preferences and include receipt of their individual genomic information as well as potential recontacting to offer participation in further studies. ${ }^{27}$ Acknowledging the challenges associated with return of genetic results, especially less-studied variants, the PMI is considering means to ensure that patients' preferences are defined in an informed fashion, they fully understand returned results, and results do not lead to unnecessary testing or interventions. ${ }^{27}$ Approaches considered by the PMI to mitigate these challenges include an effective consent process to clarify the implications of preferences, and accompanying the return of genetic results with interpretative or genomic counseling services. ${ }^{27}$ Payers are keen on addressing these challenges and have undertaken efforts to ensure that patients are informed and appropriately counseled. ${ }^{55-58}$

Participants in our study strongly recommended that qualified genetic counseling be provided not only posttesting but also pretesting, and that genetic counseling approaches and guidelines be developed to handle preferences and processes for return of multigene, mixed-validity results. The capacity, process, and cost of providing these services to PMI cohort participants may seem prohibitive, but working with private payers who have successfully tackled these issues could provide a solution. For example, several private payers have developed the capacity to provide effective and scalable pretesting and posttesting genetic counseling to their enrollees, including coverage for telephonic genetic couseling. ${ }^{59-62}$ These approaches could be leveraged by the PMI and extended to the cohort volunteers.

As the PMI solidifies the patient recontacting process, it should consider its obligation to notify participants when new research concerning their already captured alterations becomes available. Recom- mendations provided by payers in our study could be incorporated and detailed by PMI to create a robust recontacting process and enrich participants' understanding of their genomes with newly available data in an appropriate fashion.

The limitation of our study is the relatively small payer interview cohort. However, payers in our cohort have a large national impact based on their share of the US population, including the 8 largest national private payers. Their policies are monitored and often followed by other payers, and they manage an important portion of the US public plans, such as some Medicaid and Medicare Advantage plans. Although our study identified opportunities to address barriers to positive coverage of HCPs, future efforts, within and outside the PMI, to build on our findings and detail these opportunities are necessary to transform them into actionable solutions and seek consensus across payers.

\section{Conclusions}

We found barriers to insurance coverage for HCPs, including poor fit with coverage frameworks, insufficient rigor and gaps in evidence on cancer risk and actionability, and concerns about transparency to patients. Opportunities to address barriers included developing payer-suggested evidence end points that are feasible for insurance coverage; approaches to better inform patients about the potential for identifying aberrations in genes with unknown or evolving clinical validation, along with methods for recontacting patients in future; and a possibility of adjusting coverage frameworks. Our findings can inform the PMI in 3 areas: (1) addressing insurance coverage barriers to secure access to future PMI discoveries; (2) incorporating payers' evidentiary requirements into the PMI research agenda to increase relevance of research to future coverage decisions; and (3) leveraging payers' recommendations and experience to keep patients informed and engaged. Integration of these perspectives into the PMI's agenda and priorities will contribute to its success and enable ultimate access to its discoveries. 


\section{References}

1. Robson ME, Bradbury AR, Arun B, et al. American Society of Clinical Oncology policy statement update: genetic and genomic testing for cancer susceptibility. J Clin Oncol 2015;33:3660-3667.

2. Daly MB, Pilarski R, Axilbund JE, et al. Genetic/Familial high-risk assessment: breast and ovarian, version 2.2015. J Natl Compr Canc Netw 2016;14:153-162.

3. Hampel $\mathrm{H}$. NCCN increases the emphasis on genetic/familial high-risk assessment in colorectal cancer. J Natl Compr Canc Netw 2014;12(5 Suppl):829-831.

4. Remarks by the President on Precision Medicine. Available at: https:// www.whitehouse.gov/the-press-office/2015/01/30/remarks-presidentprecision-medicine. Accessed October 2, 2015.

5. FACT SHEET: President Obama's Precision Medicine Initiative. Available at: https://www.whitehouse.gov/the-press-office/2015/01/30/fact-sheetpresident-obama-s-precision-medicine-initiative. Accessed October 22, 2015.

6. Collins FS, Varmus H. A new initiative on precision medicine. N Engl J Med 2015;372:793-795.

7. Collins FS. Vision for the Cohort and the Precision Medicine Initiative. Available at: http://www.nih.gov/precisionmedicine/workshoppresentations/day-1/00-intro-collins.pdf. Accessed October 31, 2015.

8. Terry SF. Obama's Precision Medicine Initiative. Genet Test Mol Biomarkers 2015;19:113-114.

9. Hall MJ, Forman AD, Pilarski R, et al. Gene panel testing for inherited cancer risk. J Natl Compr Canc Netw 2014;12:1339-1346.

10. Kurian AW, Ford JM. Multigene panel testing in oncology practice: how should we respond? JAMA Oncol 2015;1:277-278.

11. Swisher EM. Usefulness of multigene testing: catching the train that's left the station. JAMA Oncol 2015;1:951-952.

12. Desmond A, Kurian AW, Gabree M, et al. Clinical actionability of multigene panel testing for hereditary breast and ovarian cancer risk assessment. JAMA Oncol 2015;1:943-951.

13. Kurian AW, Hare EE, Mills MA, et al. Clinical evaluation of a multiplegene sequencing panel for hereditary cancer risk assessment. J Clin Oncol 2014;32:2001-2009.

14. Yu PP, Vose JM, Hayes DF. Genetic cancer susceptibility testing: increased technology, increased complexity. J Clin Oncol 2015;33:3533-3534.

15. Kurian AW, Kingham KE, Ford JM. Next-generation sequencing for hereditary breast and gynecologic cancer risk assessment. Curr Opin Obstet Gynecol 2015;27:23-33.

16. Domchek SM, Bradbury A, Garber JE, et al. Multiplex genetic testing for cancer susceptibility: out on the high wire without a net? J Clin Oncol 2013;31:1267-1270

17. Stadler ZK, Schrader KA, Vijai J, et al. Cancer genomics and inherited risk. J Clin Oncol 2014;32:687-698.

18. Robson M. Multigene panel testing: planning the next generation of research studies in clinical cancer genetics. J Clin Oncol 2014;32:19871989.

19. Katz SJ, Kurian AW, Morrow M. Treatment decision making and genetic testing for breast cancer: mainstreaming mutations. JAMA 2015;314:997998.

20. Domchek SM. Evolution of genetic testing for inherited susceptibility to breast cancer. J Clin Oncol 2015;33:295-296.

21. Easton DF, Pharoah PD, Antoniou AC, et al. Gene-panel sequencing and the prediction of breast-cancer risk. N Engl J Med 2015;372:2243-2257.

22. Clain E, Trosman JR, Douglas MP, et al. Availability and payer coverage of BRCA1/2 tests and gene panels. Nat Biotechnol 2015;33:900-902.

23. Deverka PA, Kaufman D, McGuire AL. Overcoming the reimbursement barriers for clinical sequencing. JAMA 2014;312:1857-1858.

24. Chakradhar $S$. Insurance companies are slow to cover next-generation sequencing. Nat Med 2015;21:204-205.

25. Burns J. Health insurers struggle to manage number, cost of genetic tests. Manag Care 2016;25:20-23.

26. TRANSPERS Payer Coverage Policy Registry. University of California, San Francisco Web site. Available at: https://pharm.ucsf.edu/transpers/ node/5471. Accessed October 10, 2016.

27. The Precision Medicine Initiative Cohort Program - Building a Research Foundation for 21st Century Medicine. Precision Medicine Initiative (PMI) Working Group Report to the Advisory Committee to the Director, NIH. Available at: http://acd.od.nih.gov/reports/DRAFT-PMI-WGReport-9-11-2015-508.pdf. Accessed October 31, 2015.
28. Ritchie J, Lewis J. Qualitative Research Practice: A Guide for Social Science Students and Researchers. Thousand Oaks, CA: Sage Publications; 2003.

29. Pope C, Mays N. Qualitative Research in Health Care, 3rd ed. Malden, MA: Blackwell Publishing/BMJ Books; 2006.

30. Trosman JR, Van Bebber SL, Phillips KA. Coverage policy development for personalized medicine: private payer perspectives on developing policy for the 21-gene assay. J Oncol Pract 2010;6:238-242.

31. Van Bebber SL, Trosman JR, Liang SY, et al. Capacity building for assessing new technologies: approaches to examining personalized medicine in practice. Per Med 2010;7:427-439.

32. Weldon CB, Trosman JR, Gradishar WJ, et al. Barriers to the use of personalized medicine in breast cancer. J Oncol Pract 2012;8:e24-31.

33. Trosman JR, Weldon CB, Schink JC, et al. What do providers, payers and patients need from comparative effectiveness research on diagnostics? The case of HER2/neu testing in breast cancer. J Comp Eff Res 2013;2:461-477.

34. Trosman JR, Weldon CB, Kelley RK, Phillips KA. Challenges of coverage policy development for next-generation tumor sequencing panels: experts and payers weigh in. J Natl Compr Canc Netw 2015;13:311-318.

35. Deverka PA. Pharmacogenomics, evidence, and the role of payers. Public Health Genomics 2009;12:149-157.

36. Bombard Y, Rozmovits L, Trudeau M, et al. Access to personalized medicine: factors influencing the use and value of gene expression profiling in breast cancer treatment. Curr Oncol 2014;21:e426-433.

37. Husereau D, Marshall DA, Levy AR, et al. Health technology assessment and personalized medicine: are economic evaluation guidelines sufficient to support decision making? Int J Technol Assess Health Care 2014;30:179_ 187.

38. The Henry J. Kaiser Family Foundation. State Health Facts. Health Insurance Coverage of the Total Population. Timeframe: 2014. Available at: http://kff.org/other/state-indicator/total-population/. Accessed April 15, 2016.

39. Pear R. As Medicare and Medicaid Turn 50, Use of Private Health Plans Surges. New York Times. July 29, 2015:A12.

40. Patton MQ. Qualitative Research and Evaluation Methods, 3rd ed. Thousand Oaks, CA: Sage Publications; 2002.

41. Top 25 U.S. Health Plans, Ranked by Total Medical Enrollment (as of December 2014). AISHealth Web site. Available at: https://www.aishealth. com/health-plan-business/data/enrollment. Accessed April 15, 2016.

42. Levy DE, Byfield SD, Comstock CB, et al. Underutilization of BRCA1/2 testing to guide breast cancer treatment: black and Hispanic women particularly at risk. Genet Med 2011;13:349-355.

43. Cragun D, Bonner D, Kim J, et al. Factors associated with genetic counseling and BRCA testing in a population-based sample of young Black women with breast cancer. Breast Cancer Res Treat 2015;151:169-176.

44. Komenaka IK, Nodora JN, Madlensky L, et al. Participation of low-income women in genetic cancer risk assessment and BRCA 1/2 testing: the experience of a safety-net institution. J Community Genet 2016;7:177183.

45. CMS Official Says Whole Genome Sequencing Coverage is Unlikely. Available at: http://www.cap.org/apps/cap.portal?_nfpb=true\&cntvwrPtlt_ actionOverride $=\% 2$ Fportlets\%2FcontentViewer\%2Fshow\&_windowLab el=cntvwrPtlt\&cntvwrPtlt\% 7BactionForm.contentReference\%7D=statli ne\%2Fstat101311.html\&_state $=$ maximized\&_pageLabel=cntvwr\#Story5. Accessed September 15, 2016.

46. Toner B. Convincing CMS to Reimburse Whole-Genome Sequencing Will be "Challenging," Official Says. Available at: https://www.genomeweb. $\mathrm{com} /$ sequencing/convincing-cms-reimburse-whole-genome-sequencingwill-be-challenging-official-sa. Accessed January 4, 2017.

47. Optimizing FDA's Regulatory Oversight of Next Generation Sequencing Diagnostic Tests_Preliminary Discussion Paper. Available at: http://www. fda.gov/downloads/MedicalDevices/NewsEvents/WorkshopsConferences/ UCM427869.pdf. Accessed April 5, 2016.

48. Kracov DA, Pollard VT, Rudolf PM, Madsen JB. FDA's Evolving Framework for Oversight of Next-Generation Sequencing. Available at: http://www.arnoldporter.com/en/perspectives/publications/2015/09/fdasevolving-framework-for-oversight-of-nextgen_. Accessed April 15, 2016.

49. Evans BJ, Burke W, Jarvik GP. The FDA and genomic tests—getting regulation right. N Engl J Med 2015;372:2258-2264.

50. Trosman JR, Van Bebber SL, Phillips KA. Health technology assessment and private payers's coverage of personalized medicine. Am J Manag Care 2011;17(5 Spec No):SP53-60.

51. Lander ES. Cutting the Gordian helix-regulating genomic testing in the era of precision medicine. N Engl J Med 2015;372:1185-1186. 
52. Litwack ED, Mansfield E, Shuren J. The FDA and genetic testing. N Engl J Med 2015;372:2273-2274.

53. Center for Medical Technology Policy. Initial Medical Policy and Model Coverage Guidelines for Clinical Next Generation Sequencing in Oncology. Available at: http://www.cmtpnet.org/docs/resources/Full_ Release_Version_August_13_2015.pdf. Accessed May 2, 2016.

54. The White House. Precision Medicine Initiative: Privacy and Trust Principles. November 9, 2015. Available at: https://www.whitehouse. gov/sites/default/files/microsites/finalpmiprivacyandtrustprinciples.pdf. Accessed May 2, 2016.

55. Armstrong J, Toscano M, Kotchko N, et al. Utilization and outcomes of BRCA genetic testing and counseling in a national commercially insured population: the ABOUT study. JAMA Oncol 2015;1:1251-1260.

56. Sandy LG, Tuckson RV, Stevens SL. UnitedHealthcare experience illustrates how payers can enable patient engagement. Health Aff (Millwood) 2013;32:1440-1445.

57. Wang G, Beattie MS, Ponce NA, Phillips KA. Eligibility criteria in private and public coverage policies for BRCA genetic testing and genetic counseling. Genet Med 2011;13:1045-1050.
58. Graf MD, Needham DF, Teed N, Brown T. Genetic testing insurance coverage trends: a review of publicly available policies from the largest US payers. Per Med 2013;10:235-243.

59. Aetna to Offer Access to Confidential Telephonic Cancer Genetic Counseling to Health Plan Members. Available at: http://www. businesswire.com/news/home/20071127006263/en/Aetna-Offer-AccessConfidential-Telephonic-Cancer-Genetic. Accessed May 2, 2016.

60. Sutphen R, Davila B, Shappell H, et al. Real world experience with cancer genetic counseling via telephone. Fam Cancer 2010;9:681-689.

61. InformedDNA Collaborates with Cigna on New Whole Exome Sequencing Policy. Available at: http://www.informeddna.com/images/news_articles/ WESpolicyCigna_PressRelease.pdf. Accessed May 2, 2016.

62. New BRCA Genetic Counseling Requirement Frequently Asked Questions. Available at: https://www.unitedhealthcareonline.com/ ccmcontent/ProviderII/UHC/en-US/Assets/ProviderStaticFiles/ ProviderStaticFilesPdf/Clinician\%20Resources/Oncology \% 20 Specialty\%20Pharmacy\%20Network/BRCA_Genetic_Counseling_ Requirement_FAQ.pdf. Accessed May 2, 2016.

\section{See JNCCN.org for supplemental online content.}

